\title{
Embarazo molar cervical. Diagnóstico y tratamiento quirúrgico con legrado uterino y ligadura de arterias cervicales
}

\section{Cervical molar pregnancy. Diagnosis and surgical treatment with uterine curettage and cervical arteries ligation}

\section{Sergio Leible ${ }^{1 *}$, Francisco Figueroa², Gabriel Mitelman³, Patricio Venegas ${ }^{3}$ y Josue Carvajal ${ }^{3}$}

${ }^{1}$ Facultad de Medicina, Universidad de Chile, Normandía Diagnóstico por Imágenes; ${ }^{2}$ Departamento de Medicina, Universidad Mayor; ${ }^{3}$ Servicio de Ginecología y Obstetricia, Hospital Clínico Félix Bulnes. Santiago de Chile, Chile

\section{Resumen}

Reportamos el caso de una mujer de 28 años con atraso menstrual de 14 días, diagnosticada en el servicio de urgencia obstétrica del Hospital Félix Bulnes con un embarazo ectópico cervical mediante ultrasonido, en contexto de metrorragia grave. El tratamiento consistió en legrado uterino segmentario más ligadura de arterias cervicales. El estudio histopatológico reveló una mola hidatiforme parcial en el producto del curetaje. La paciente evolucionó favorablemente sin requerir más intervenciones. Este caso da cuenta del exitoso manejo de un embarazo cervical con tratamiento quirúrgico, dando una oportunidad de preservar la fertilidad de la paciente.

Palabras clave: Embarazo cervical. Legrado uterino. Mola hidatiforme parcial.

\section{Abstract}

We are reporting the case of a 28-year-old woman with 14-day menstrual delay diagnosed, in the obstetric emergency department of Félix Bulnes Hospital, with a cervical pregnancy through ultrasound, in the context of severe metrorrhagia. The treatment consisted in uterine curettage and ligation of cervical arteries. A histopathological study revealed a partial hydatidiform mole in the curettage product. The patient evolved favorably without other interventions. This case its an example of the successful management of a cervical pregnancy with surgical treatment, giving a chance of preserving the fertility of the patient.

Key words: Cervical pregnancy. Uterine curettage. Partial hydatiform mole.

\section{Introducción}

El embarazo ectópico es aquel en que el blastocisto en desarrollo se implanta en un sitio distinto al endometrio de la cavidad uterina'. Aproximadamente el $96 \%$ de los embarazos ectópicos ocurren en la trompa de Falopio, pero son posibles otras localizaciones². El embarazo ectópico cervical da cuenta de menos del $1 \%$ de los embarazos ectópicos ${ }^{2}$ y ocurre cuando el saco gestacional se implanta en el revestimiento del canal endocervical ${ }^{3}$, siendo su incidencia estimada entre 1:2500 y 1:12.000 embarazos ${ }^{4}$. El diagnóstico y el tratamiento temprano de esta condición son importantes debido al alto riesgo de hemorragia grave, y prevenir la histerectomía como tratamiento resolutivo es de máxima prioridad.

Aún más infrecuente que el embarazo cervical es el embarazo molar con implantación cervical. El embarazo molar forma parte de las enfermedades
Correspondencia:

*Sergio Leible

E-mail: sergioleible@gmail.com

DOI: 10.24875/RECHOG.M21000028
Disponible en internet: 23-12-2021 Rev Chil Obstet Ginecol. 2021;86(5):465-469

www. rechog.com CC BY-NC-ND (https://creativecommons.org/licenses/by-nc-nd/4.0/). 
trofoblásticas gestacionales y su incidencia en las distintas regiones del mundo es variable, reportándose en el medio latinoamericano incidencias de 23 a 465 casos por cada 100.000 embarazos $^{5}$. Se considera una condición premaligna por su capacidad de progresar hacia neoplasias trofoblásticas gestacionales.

El embarazo ectópico cervical es una patología a la que antiguamente se le describió una mortalidad cercana al $50 \%$ cuando se practicaba una histerectomía como medida terapéutica ${ }^{6}$. Actualmente, gracias al diagnóstico y el tratamiento precoz, su mortalidad ha disminuido hasta llegar a ser menor del $1 \%{ }^{7}$. La causa del embarazo ectópico cervical no ha sido determinada, pero se cree que existe una asociación entre la instrumentalización cervicouterina ${ }^{8}$, los tratamientos de reproducción asistida ${ }^{9}$, el rápido descenso de un huevo fertilizado o un endometrio no receptivo ${ }^{3}$.

El método diagnóstico de referencia es la ecografía transvaginal, siendo sus criterios diagnósticos una cavidad uterina vacía, un cérvix engrosado en forma de barril, un saco gestacional presente bajo el nivel del orificio cervical interno, la ausencia de sliding sign (deslizamiento del saco gestacional a través del canal endocervical al presionar con el transductor cuando se trata de un aborto en evolución) y un moderado flujo alrededor del saco gestacional en el examen Doppler a color. Durante el proceso diagnóstico se debe tomar una muestra para determinación de la gonadotropina coriónica humana beta $(\beta \mathrm{HCG})^{10}$.

Respecto al tratamiento, no existen criterios establecidos acerca de qué pacientes cursarán con terapia médica o quirúrgica. El metotrexato a dosis de 0,5-1 $\mathrm{mg} / \mathrm{kg}$ debería considerarse como tratamiento de primera línea en las pacientes hemodinámicamente estables, y la terapia quirúrgica debería reservarse para las pacientes inestables o aquellas en quienes el enfoque conservador no es suficiente ${ }^{10,11}$. Sobre el manejo conservador, este se asocia con una mayor tasa de falla en casos de edad gestacional > 9 semanas, presencia de latidos cardiofetales, longitud corona-cóccix > $10 \mathrm{~mm}$ y valores de $\beta \mathrm{HCG}>10.000^{11}$; en estos casos se debe considerar el tratamiento quirúrgico, cuyas opciones pueden ser desde curetaje endocervical, embolización de las arterias uterinas o hasta histerectomía, dependiendo de la evolución de la paciente $^{10}$.

A continuación damos cuenta del caso de una paciente que consultó al servicio de ginecología y obstetricia del Hospital Félix Bulnes por metrorragia y se le diagnosticó un embarazo cervical con mola incompleta.

\section{Caso clínico}

Paciente de 28 años, G2P0A1, con antecedente de infertilidad secundaria y síndrome de Asherman posiblemente secundario al legrado uterino de un aborto espontaneo previo, que consulta por un cuadro de metrorragia grave y atraso menstrual de 14 días, test de embarazo positivo y sin antecedentes de procedimientos de fertilización asistida. En la exploración física inicial se encuentra hemodinámicamente estable, sin dolor abdominal, pálida, con la cavidad vaginal con abundantes coágulos, el orificio cervical interno cerrado y, al tacto vaginal, un útero de tamaño y forma normales. En el examen ecográfico no se observa saco gestacional en la cavidad endometrial y se reporta la siguiente descripción: «En el tercio superior de la pared posterior del canal cervical se observa imagen ovalada hipoecogénica con doble halo que mide $9,1 \times 4,8 \times 5,7 \mathrm{~mm}$. Su límite inferior está a $21 \mathrm{~mm}$ del orificio cervical externo y su límite superior a 16,5 mm de la cavidad endometrial (Fig. 1). Presenta abundante irrigación sanguínea en su periferia con vasos de flujo arterial de muy baja resistencia (IP 0,2) con una velocidad máxima de $13 \mathrm{~cm} / \mathrm{s}$. Ambos ovarios de aspecto normal y sin líquido libre en el fondo de saco de Douglas. Planteándose la hipótesis diagnóstica de embarazo cervical y metrorragia severa". Se envía a urgencias con una gasa compresiva vaginal, donde se determinan HCG con un valor de $9888 \mathrm{mUl} / \mathrm{ml}$, hematocrito del 39\% y hemoglobina de 13,9. Ingresa a pabellón con metrorragia grave sin compromiso hemodinámico. Se realiza ligadura de las arterias cervicales con puntos paracervicales y luego legrado uterino, con lo que cede completamente la metrorragia. Es dada de alta en buenas condiciones a las 48 horas.

El estudio histopatológico del material obtenido del legrado uterino dio como resultado embarazo de mola hidatiforme parcial. Se observó un rápido descenso de los valores de la HCG en plasma durante los días posteriores a la intervención quirúrgica (Fig. 2), llegando a desaparecer en el control realizado a los 52 días posteriores al tratamiento.

\section{Discusión}

El embarazo molar cervical es extremadamente infrecuente, siendo este el quinto caso reportado en la literatura $^{12,13}$. En una revisión reciente de 254 embarazos cervicales se encontró que el 35\% fueron tratados con tratamiento médico, el $28 \%$ con tratamiento 


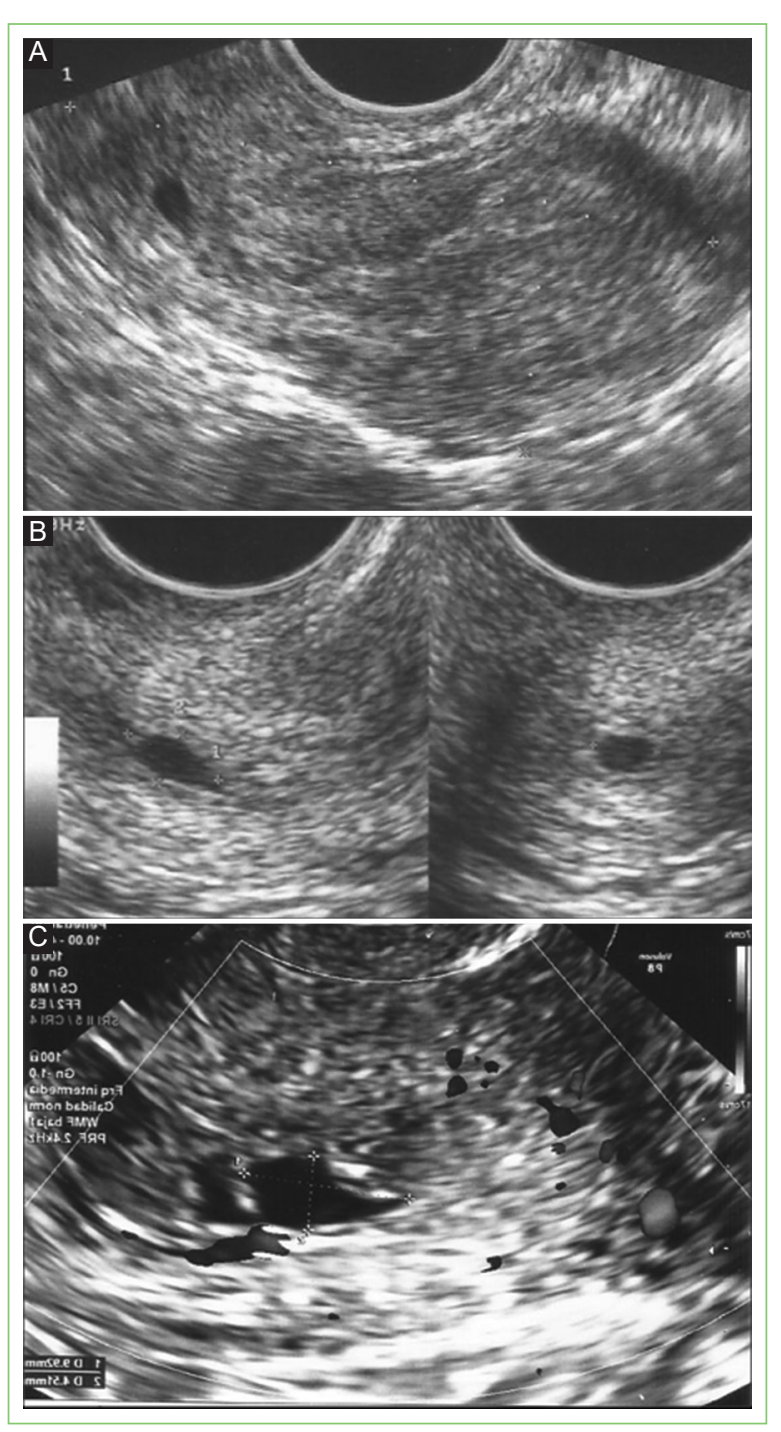

Figura 1. Imagen sacular en la pared posterior del cérvix. A: corte longitudinal de útero que muestra un saco gestacional de $9 \times 5 \times 6 \mathrm{~mm}$ en la pared posterior del cuello uterino. B: detalles del saco gestacional cervical en cortes transversal y longitudinal. C: detalle del saco gestacional cervical unas horas después del primer examen.

quirúrgico y el $38 \%$ combinando ambos tipos de tratamiento. Un alto índice de sospecha, junto con una revisión meticulosa de los hallazgos clínicos y radiológicos, son esenciales para hacer un diagnóstico preciso del embarazo cervical. El éxito del tratamiento conservador depende principalmente del diagnóstico precoz $^{14}$.

El diagnóstico de embarazo cervical suele hacerse por ecografía transvaginal al observar un saco gestacional con embrión implantado en las paredes del cuello uterino ${ }^{11}$. Sin embargo, si no hay estructuras embrionarias dentro del saco, como saco vitelino o embrión, el diagnóstico se hace más dificultoso, ya que la imagen sacular puede confundirse con un quiste de Naboth, estructura muy frecuente de observar en el cuello uterino en mujeres en edad fértil ${ }^{15}$. Para el diagnóstico diferencial fue muy útil en este caso la imagen obtenida con Doppler $\operatorname{color}^{16}$ y la onda espectral de velocidades arteriales obtenida con Doppler pulsado de los vasos sanguíneos localizados alrededor del saco gestacional (Fig. 3), ya que esta imagen con vasos arteriales de bajo flujo y baja resistencia alrededor del saco es propia del embarazo y no suele observarse alrededor de un quiste de Naboth, y es más específica, según nuestra opinión, que la imagen de doble halo, que en este caso también estaba presente, pero que requiere cierta subjetividad para su interpretación. El otro elemento ecográfico a tener en cuenta en este caso fue la ausencia de signos sugerentes de restos ovulares, a saber, contenido hiperrefringente usualmente de forma irregular con una o más arterias espirales dilatadas, en alrededor de $2 \mathrm{~mm}$ de diámetro, en la base de implantación ${ }^{17}$. Resumiendo, ante un cuadro de metrorragia grave con HCG plasmática presente, sin saco gestacional ni restos ovulares al examen ecográfico transvaginal, y con una imagen sacular vacía con abundante irrigación en su periferia en el cérvix, se planteó el diagnóstico de embarazo cervical.

Hecho el diagnóstico, y debido a la presencia de metrorragia grave que no cedió con compresas vaginales, el equipo de turno decidió realizar tratamiento quirúrgico conservador considerando la edad y el deseo de paridad de la paciente. La ligadura de las ramas cervicales de las arterias uterinas para el tratamiento del embarazo cervical fue descrita por primera vez en 1983 por Ratten ${ }^{18}$, y es una alternativa que en este caso resultó adecuada para la pronta cesación del cuadro de metrorragia grave, permitiendo un alta precoz y en buenas condiciones de la paciente.

Un diagnóstico oportuno y un tratamiento quirúrgico conservador permitieron en este caso preservar la fertilidad de la paciente. La tasa de mortalidad en las pacientes con embarazo cervical es alta, alrededor de un $1 \%^{6}$, y la tasa de morbilidad con compromiso futuro de la fertilidad suele ser aún mayor ${ }^{15}$. La ligadura de las arterias cervicales ${ }^{19}$ junto con legrado cervical ${ }^{7} y$ balón compresivo ${ }^{20}$ son las técnicas que se han descrito en la literatura como tratamiento conservador del embarazo cervical, y deben tenerse presentes, como en este caso, ante un diagnóstico de posible embarazo 


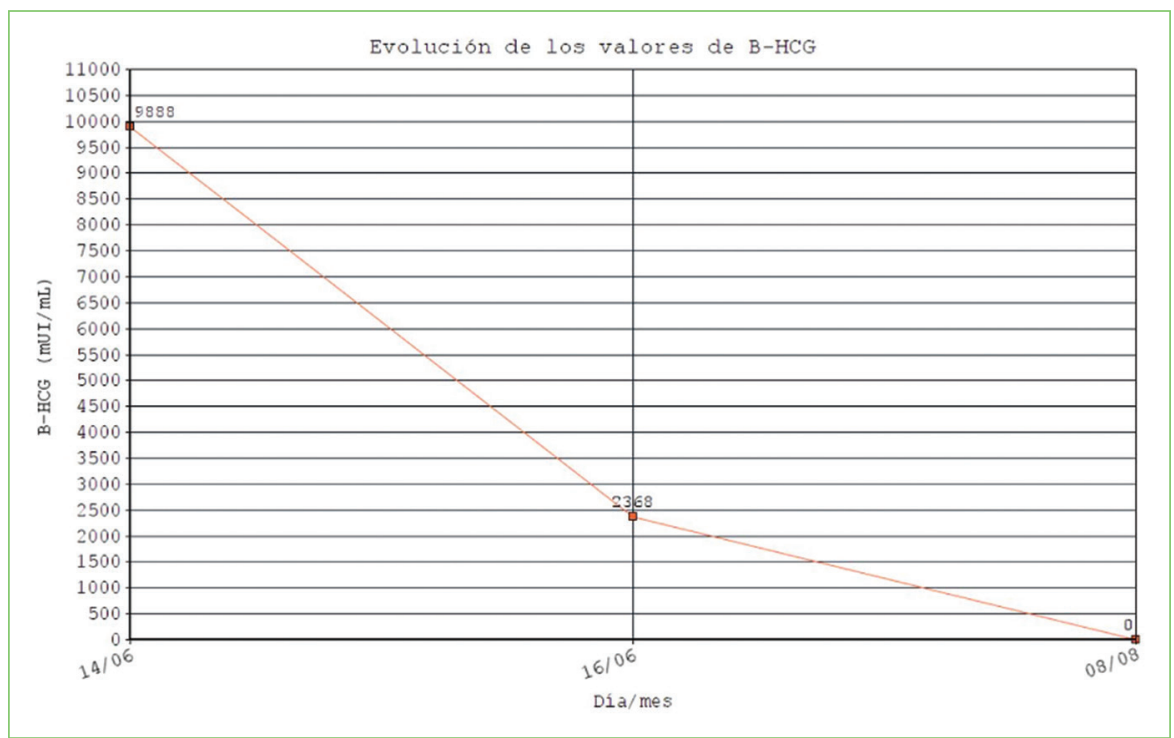

Figura 2. Curva de los valores de gonadotrofina coriónica humana.

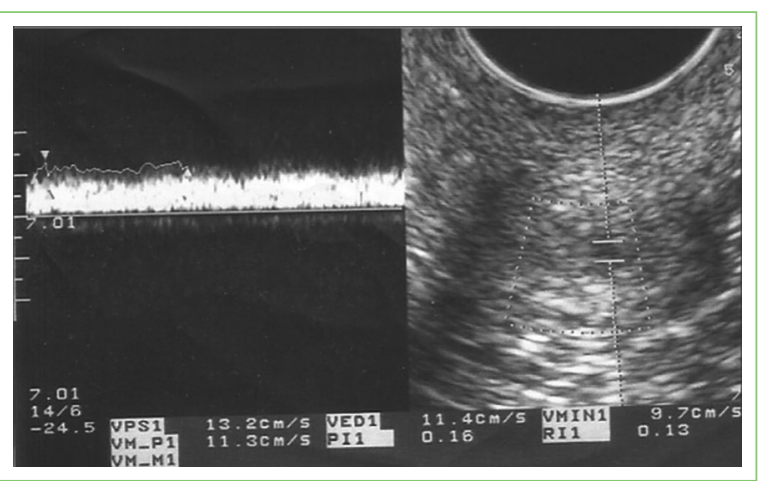

Figura 3. Onda espectral de los vasos sanguíneos arteriales de baja resistencia alrededor del saco gestacional cervical.

cervical y metrorragia grave. En esta oportunidad no fue necesario adjuntar terapia con metotrexato ${ }^{11}$ debido al rápido descenso de la curva de HCG.

\section{Financiamiento}

Este artículo no cuenta con ninguna fuente de financiamiento.

\section{Conflicto de intereses}

Los autores del artículo no declaran ningún conflicto de intereses.

\section{Responsabilidades éticas}

Protección de personas y animales. Los autores declaran que para esta investigación no se han realizado experimentos en seres humanos ni en animales.

Confidencialidad de los datos. Los autores declaran que han seguido los protocolos de su centro de trabajo sobre la publicación de datos de pacientes.

Derecho a la privacidad y consentimiento informado. Los autores han obtenido el consentimiento informado de los pacientes y/o sujetos referidos en el artículo. Este documento obra en poder del autor de correspondencia.

\section{Bibliografía}

1. Tulandi T. Ectopic pregnancy: epidemiology, risk factors, and anatomic sites. UpToDate; 2019. (Consultado el 24/10/2019.) Disponible en: https://www.uptodate.com/contents/ectopic-pregnancy-methotrexate-therapy?search=ectopic-pregnancy-epidemiology-risk-factors-and-anatomic-\&source=search_result\&selectedTitle=6 150\&usage_type=default\&display_rank $=6$

2. Bouyer J, Coste J, Fernández H, Pouly JL, Job-Spira N. Sites of ectopic pregnancy: a 10-year population-based study of 1800 cases. Hum Reprod. 2002; 17:3224-30

3. Tulandi T. Cervical pregnancy. UpToDate; 2019. (Consultado el 24/10/2019.) Disponible en: https://www.uptodate.com/contents/cervical-pregnancy

4. Epni I, Ocal P, Erkan S, Erkiz B. Conservative treatment of cervical ectopic pregnancy with transvaginal ultrasound guided aspiration and single-dose methotrexate. Fertil Steril. 2004:81:1130-2.

5. Altieri A, Franceschi S, Ferlay J, Smith J, Vecchia CL. Epidemiology and aetiology of gestational trophoblastic diseases. Lancet Oncol. 2003;4:670-8.

6. Río de la Loza Cava L, Moyers-Arévalo JA. Embarazo ectópico cervical. Cuando el tratamiento conservador falla. Reporte de un caso y revisión de la bibliografía. Ginecol Obstet Mex Mex. 2012;80:668-72. 
7. Troncoso F, Cardone X, Rondini C, Troncoso C. Embarazo ectópico cervical: diagnóstico y tratamiento conservador con cerclaje cervical. Rev Chil Obstet Ginecol. 2005;70:257-60.

8. Ushakov FB, Elchalal U, Aceman PJ, Schenker JG. Cervical pregnancy: past and future. Obstet Gynecol Surv. 1997;52:45-59.

9. Karande VC, Flood JT, Heard N, Veeck L, Muasher SJ. Analysis of ectopic pregnancies resulting from in-vitro fertilization and embryo transfer. Hum Reprod. 1991;6:446-9.

10. Vergara P, Sepúlveda A, Parra M. Embarazo ectópico cervical y cicatriz de cesárea. Diagnóstico y manejo. Rev Hosp Clin Univ Chile. 2019;30:57-70.

11. Cipullo L, Cassese S, Fasolino L, Fasolino M, Fasolino A. Cervical pregnancy: a case series and a review of current clinical practice. Eur $J$ Contracept Reprod Health Care. 2008;13:313-9.

12. Aytan $\mathrm{H}$, Caliskan AC, Demirturk F, Koseoglu RD, Acu B. Cervical partia hydatidiform molar pregnancy. Gynecol Obstet Invest. 2008;66:142-4.

13. Schwentner L, Schmitt W, Bartusek G, Kreienberg R, Herr D. Cervical hydatidiform mole pregnancy after missed abortion presenting with severe vaginal bleeding: case report and review of the literature. Eur J Obstet Gynecol Reprod Biol. 2011;156:9-11.
14. Hosni MM, Herath RP, Mumtaz R. Diagnostic and therapeutic dilemmas of cervical ectopic pregnancy. Obstet Gynecol Surv. 2014;69:261-76.

15. Fogel SR, Slasky BS. Sonography of Nabothian cysts. AJR Am J Roentgenol. 1982;138:927-30.

16. Pellerito JS, Taylor KJ, Quedens-Case C, Hammers LW, Scoutt LM, Ramos IM, et al. Ectopic pregnancy: evaluation with endovaginal color flow imaging. Radiology. 1992;183:407-11.

17. Atri M, Rao A, Boylan C, Rasty G, Gerber D. Best predictors of grayscale ultrasound combined with color Doppler in the diagnosis of retained products of conception. J Clin Ultrasound. 2011;39:122-7.

18. Ratten GJ. Cervical pregnancy treated by ligation of the descending branch of the uterine arteries. Case report. Br J Obstet Gynaecol. 1983;90:367-71.

19. Viera M, Molina L, Tapia G. Embarazo ectópico cervical. Rev Cuba Obstet Ginecol. 2017;43:125-35.

20. Ramírez J, Pimentel J, Cabrera S, Campos G, Zapata, B. Embarazo heterotópico cervical: reporte de un caso. Rev Peru Ginecol Obstet. 2015;61:301-6. 